Mens

revue d'histoire intellectuelle de l'Amérique française

Site Internet du Centre de recherche en civilisation canadienne-française (CRCCF), http://www.crccf.uottawa.ca/

\title{
Marie LeBel
}

Volume 7, numéro 2, printemps 2007

URI : https://id.erudit.org/iderudit/1024137ar

DOI : https://doi.org/10.7202/1024137ar

Aller au sommaire du numéro

Éditeur(s)

Centre de recherche en civilisation canadienne-française

ISSN

1492-8647 (imprimé)

1927-9299 (numérique)

Découvrir la revue

Citer ce compte rendu

LeBel, M. (2007). Compte rendu de [Site Internet du Centre de recherche en civilisation canadienne-française (CRCCF), http://www.crccf.uottawa.ca/]. Mens, 7(2), 369-372. https://doi.org/10.7202/1024137ar d'utilisation que vous pouvez consulter en ligne.

https://apropos.erudit.org/fr/usagers/politique-dutilisation/ 


\section{Sites INTERNET}

\section{Site Internet du Centre de recherche en civilisation canadienne-française (CRCCF), http://www.crccf.uottawa.ca/}

Le Centre de recherche en civilisation canadienne-française (CRCCF) a été fondé en 1958 par quatre professeurs du Département de français de l'Université d'Ottawa afin de développer l'enseignement des lettres canadiennes-françaises au niveau universitaire. Si le partenariat avec l'Université d'Ottawa se poursuit, le site Internet officiel de l'institution hébergeant celui du Centre; le mandat de celui-ci a quant à lui été considérablement élargi. À la littérature, se sont ajoutées d'autres disciplines qui contribuent à une meilleure compréhension du Canada français : linguistique, histoire, sociologie, etc. En outre, cette diversification fait du centre de recherche, et par ricochet de son site Internet, l'exemple d'une réussite de l'interdisciplinarité.

Le site Internet témoigne de la maturité acquise par le Centre et de sa capacité à diffuser ses ressources à mesure que se renouvèlent les modes de diffusion des connaissances. On y constate aussi le dynamisme de l'organisme, puisque ne cessent de s'ajouter des partenariats et que s'élargit le réseau des collaborateurs.

Plusieurs projets de recherche chapeautés par le CRCCF se côtoient dans le site. C'est leur multidisciplinarité qui frappe. Linguistes, historiens, littéraires y fréquentent les sociologues et les spécialistes de l'éducation dans des projets originaux et intéressants. Une lecture rapide nous apprend que dans le domaine historique, Jean-Pierre Wallot dirige une vaste recherche sur le Bas-Canada au XIX ${ }^{\mathrm{e}}$ siècle. En littérature, on découvre le projet Biblifo de Lucie Hotte. Paul Wyczynski et 
Yolande Grisé, qui dirigent quant à eux une édition critique des œuvres complètes de François-Xavier Garneau, y sont présents. Un groupe s'attache à informatiser le Dictionnaire de l'Amérique française. Enfin, le CRCCF, dépositaire de quelque 2500 manuels scolaires, offre une base de données (MASCOFO) sur les manuels scolaires (1850-1995).

La recension que nous avons effectuée porte cependant essentiellement sur les expositions virtuelles « 400 ans de présence française au Canada, 1604-2004 » et "Mémoire de la francophonie canadienne: documents historiques conservés au CRCCF ». Ces rubriques mettent en valeur la présence française en Amérique et insistent pour faire remonter les origines de la francophonie ontarienne au tout début du XVII ${ }^{\mathrm{e}}$ siècle. En ce sens, il apparait que l'interprétation historique retrouvée dans les expositions virtuelles du CRCCF annonce une volonté certaine des historiens de l'Ontario français de fournir aux francophones de cette province un récit «national» s'inscrivant dans la longue durée. On a ainsi le sentiment d'assister aux balbutiements d'une tradition historiographique cherchant à poser les jalons et les figures d'un récit historique à fonction identitaire. Ces figures historiques - largement puisées au répertoire canadien-français que les auteurs des expositions sentent remis en cause par les configurations et les reconfigurations d'un récit à dominante québécoise - posent des bornes identitaires qui renouent, plus ou moins heureusement, avec le passé d'un Canada français revu et corrigé à l'ontarienne. L'exposition virtuelle « 400 ans de présence française au Canada, 1604-2004 » confirme tout particulièrement une nette visée historiographique dans ce qu'elle rejoint, par ses objectifs et son contenu, le premier manuel d'histoire de l'Ontario français publié en 2004 sous l'égide du Centre franco-ontarien de ressources pédagogiques. Les figures présentées, dépoussiérées et rajeunies, sont les Champlain, 
Étienne Brûlé, Desgroseillers, Radisson, Dollard des Ormeaux. Ces personnages ponctuent le récit construit ; les textes ont un ton académique aux qualités didactiques indéniables. Le récit se veut fondateur de l'exploration et de l'enracinement des francophones dans les "Pays d'en-Haut». Les faits et personnages retenus et commentés et le traitement qui leur est réservé contribuent tous à cet objectif de fondation. Le paradigme qui oriente le récit proposé nous semble double et complémentaire. Il s'agit de montrer une communauté d'explorateurs qui s'est adaptée au fil des siècles à la culture et à la langue de l'autre - tour à tour celle des Premières Nations et celle des conquérants anglophones - sans perdre ni sa culture ni sa langue ; une communauté qui a lutté pour s'adapter aux institutions de la majorité ; une communauté qui s'accommode du système politique de la majorité, en le comprenant et en s'en servant.

Reste à savoir si ce récit, et le paradigme qui le soustend, sont porteurs d'avenir pour une communauté qui est bien plus diversifiée et complexe que ne le suggère un récit univoque. Il faut bien l'admettre, au-delà du survol très politiquement correct de la fin du $\mathrm{XX}^{\mathrm{e}}$ siècle - à saveur d'accommodement face à la majorité anglophone et d'acceptation de la diversité culturelle qui constitue désormais la francophonie ontarienne - la congruence entre le passé proposé et l'avenir espéré n'est pas évidente.

Il faut déplorer les dangers du support de communication emprunté. En effet, le site Internet exige d'assurer également la mise à jour des informations pour l'ensemble des composantes présentées. Relativement au site recensé, on notera que certains projets sont régulièrement mis à jour - les expositions du CRCCF et Biblifo, notamment. Pour d'autres, malheureusement, les dernières mises à jour remontent à 2001. Les expositions virtuelles, nous le précisons, ne pâtissent pas 
de ce travers. Cependant, le visiteur peut mettre en doute la rectitude de certaines informations eu égard à l'inégalité de traitement des composantes du site.

Néanmoins, le site du CRCCF se distingue en ce qui a trait à l'expertise et au contenu. Les collaborateurs sont des chercheurs et des spécialistes dans les disciplines qui contribuent aux divers projets de recherche. Ils sont nombreux et qualifiés : linguistes, historiens, sociologues ou folkloristes. La banque de données documentaires est en outre extrêmement riche. La carte du site est complète et lisible. Le travail d'édition est quasi irréprochable, ce qui n'est pas négligeable. Le travail d'informatisation d'une banque d'informations extrêmement riche est amorcé sans être complété. Ainsi, en date de la recension, le chercheur risque d'aboutir à un cul-de-sac décevant selon le document qu'il cherche à consulter. Toutefois, il trouvera dans la rubrique des expositions virtuelles le régime d'historicité qui semble s'enclencher en Ontario français, la trame d'un récit historique pensé (repensé) dans la longue durée, la réappropriation de figures historiques susceptibles d'instrumentaliser ce récit.

Marie LeBel

Histoire

Collège universitaire de Hearst 